\title{
Solvent effects on 2-methoxyethanol conformers: an ab initio DFT study using the SCI-PCModel
}

\author{
Francisco P.S.C. Gil ${ }^{\mathrm{a}, *}$, J.J.C. Teixeira-Dias ${ }^{\mathrm{b}}$ \\ ${ }^{a}$ Department of Physics, University of Coimbra, P-3049 Coimbra, Portugal \\ ${ }^{\mathrm{b}}$ Department of Chemistry, University of Aveiro, P-3810 Aveiro, Portugal
}

\begin{abstract}
The four stable conformers of 2-methoxyethanol, $\mathrm{CH}_{3} \mathrm{O}-\mathrm{CH}_{2}-\mathrm{CH}_{2}-\mathrm{OH}\left(\mathrm{tgg}^{\prime}, \mathrm{ggg}{ }^{\prime}, \mathrm{ttg}\right.$ and ttt) are studied by ab initio calculations using the SCRF theory and the SCI-PCModel to assess solvent effects on the structure and vibrational spectra of this compound. Full geometry optimizations were carried out at the B3LYP/6-311+G(3df,2df,2p)//B3LYP/6-31G(d) level, and normal mode calculations were performed within the harmonic approximation using the B3LYP/6-31G(d) derived force fields. The solvent, herein represented by a polarizable continuum with a defined dielectric constant, is found to affect significantly the geometry and dipole moment of the individual solute conformers. In addition, the results seem to suggest that the intramolecular H-bond type of interactions, which occur for $\operatorname{tgg}^{\prime}$ and $g g g^{\prime}$, tend to attenuate the solvent influence. In turn, the conformers which do not exhibit intramolecular H-bond interactions, ttg and ttt, exhibit a stronger solvent influence as revealed mainly by structural and electrostatic changes affecting both the ether O atom and the hydroxyl group. (C) 1999 Elsevier Science B.V. All rights reserved.
\end{abstract}

Keywords: 2-methoxyethanol; solvent effects; Self-Consistent Reaction Field (SCRF); Self-Consistent Isodensity Polarizable Continuum Model (SCI-PCM)

\section{Introduction}

2-Methoxyethanol $\left(\mathrm{CH}_{3} \mathrm{OCH}_{2} \mathrm{CH}_{2} \mathrm{OH}\right.$, hereafter abbreviated as 2ME) has three important conformational degrees of freedom involving the $\mathrm{CO}-\mathrm{CC}, \mathrm{OC}-$ $\mathrm{CO}$ and $\mathrm{CC}-\mathrm{OH}$ dihedral angles. The two more stable conformations of this molecule, $\operatorname{tgg}^{\prime}$ and $\mathrm{ggg}^{\prime}$, owe most of their stability to a conjugation of conformational preferences about these dihedral angles, the occurrence of an intramolecular $\mathrm{O} \cdots \mathrm{H}-\mathrm{O}$ hydrogen bond interaction, and $\mathrm{H} \cdots \mathrm{H}$ steric repulsive

\footnotetext{
* Corresponding author. Tel: + 35139 410629, fax: + 35139 829158.

E-mail address: fgil.gemini.ci.uc.pt (F.P.S.C. Gil)
}

interactions. For the isolated molecule situation, $\operatorname{tgg}^{\prime}$ has been found to be more stable than $\mathrm{ggg}^{\prime}[1-3]$. For none of the above mentioned rotors $(\mathrm{CO}-\mathrm{CC}, \mathrm{OC}-$ $\mathrm{CO}$ and $\mathrm{CC}-\mathrm{OH}$ ) does the $2 \mathrm{ME}$ molecule act rigidly, as the skeletal bond angles and the dihedral angles change appreciably with conformation.

In the liquid phase, FTIR and Raman spectra of 2ME show features ascribed to different degrees of aggregation through intermolecular hydrogen bonding [1]. In particular, the formation of stable intermolecular hydrogen bonded dimers is thought to play a role on the structure and dynamics of $2 \mathrm{ME}$ in the liquid phase [4].

In the present work, non-specific interactions between the solute molecule, as represented by each of the $2 \mathrm{ME}$ conformers, and the solvent, as 


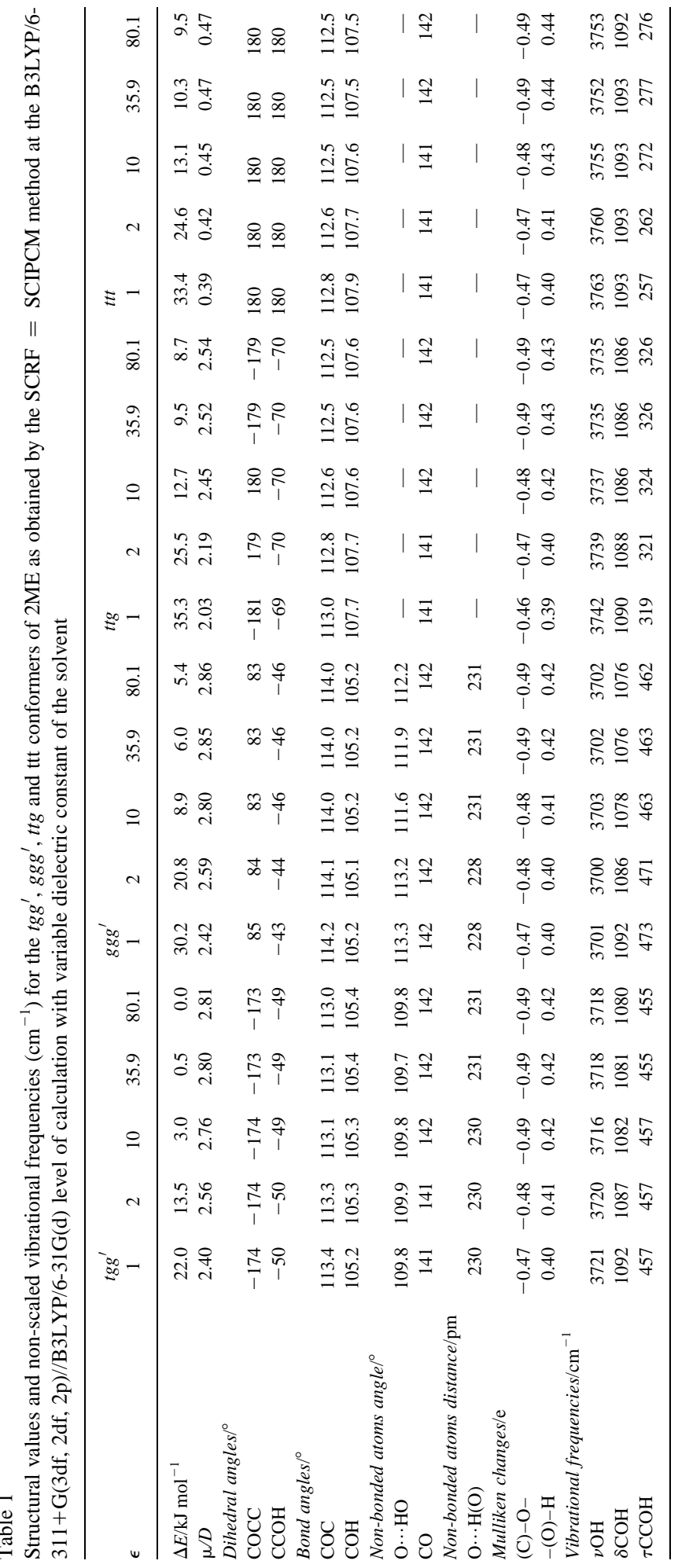




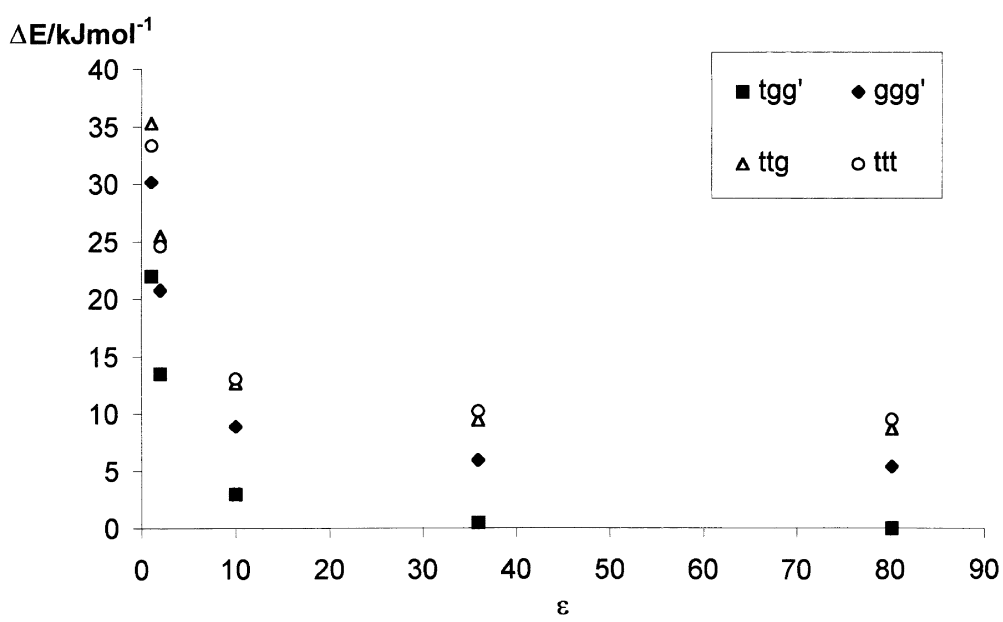

Fig. 1. Energy differences $\left(\Delta E / \mathrm{kJ} \mathrm{mol}^{-1}\right)$ for the four more stable conformers of $2 \mathrm{ME}$ obtained by the SCRF $=\mathrm{SCIPCM}$ method at the B3LYP/ 6-311+G (3df, 2df, 2p)//B3LYP/6-31G(d) level of calculation with variable dielectric constant of the solvent.

represented by a polarizable continuum with a particular dielectric constant, are considered within the scope of the Self Consistent Reaction Field (SCRF) theory, using the Self Consistent Isodensity Polarizable Continuum Model (SCI-PCM). The main question addressed in this work is "to what extent is the solvent as a continuum affecting the relative stability, structure and vibrational frequencies of the $2 \mathrm{ME}$ conformers?". In particular, "what is the solvent influence on the intramolecular hydrogen bond interactions in some of the $2 \mathrm{ME}$ conformers $\left(\operatorname{tgg}^{\prime}\right.$ and $\left.g g g^{\prime}\right)$ ?'”. These questions will be herein addressed within the framework of Density Functional theory, at the B3LYP/6-311+G(3df,2df,2p)//(B3LYP/6$31 \mathrm{G}(\mathrm{d})$ level of calculation, with the normal mode calculations being performed within the harmonic approximation, using the B3LYP/6-31G(d) derived force fields. In having selected $2 \mathrm{ME}$ as the solute system, it is understood that $2 \mathrm{ME}$ is a particularly good model system for assessing solvent effects, mainly because some of its conformers are stabilized by intramolecular hydrogen bond type of interactions.

\section{Computational methods}

Each one of the 2ME conformers herein considered is identified by a three-letter acronym specifying the $\mathrm{CO}-\mathrm{CC}, \mathrm{OC}-\mathrm{CO}$ and $\mathrm{CC}-\mathrm{OH}$ axes as trans $(\mathrm{t})$, + gauche $(\mathrm{g})$ or - gauche $\left(\mathrm{g}^{\prime}\right)$ arrangements. For the identification of the atoms in structural parameters, a left-to-right order of the italicized atomic symbols in $\mathrm{C}\left(\mathrm{H}_{3}\right) \mathrm{OC}\left(\mathrm{H}_{2}\right) \mathrm{C}\left(\mathrm{H}_{2}\right) \mathrm{OH}$ is adopted.

$\mathrm{Ab}$ initio calculations were carried out at the B3LYP/6-311+G(3df,2df,2p)//B3LYP(6-31G(d) level, within the framework of SCRF (Self-Consistent Reaction Field) theory, using the SCI-PCModel (Self-Consistent Isodensity Polarized Continuum Model) $[5,6]$. The GAUSSIAN 94W program package [7] was used in the calculations. Specific interactions between $2 \mathrm{ME}$ and the solvent molecules were not considered in this study, since the solvent is represented by a polarizable continuum with a particular dielectric constant, $\epsilon$. The absolute errors in bond lengths and bond angles with respect to the equilibrium geometrical parameters are less than $1 \mathrm{pm}$ and $0.1^{\circ}$, respectively. Calculations of vibrational frequencies were carried out using the above mentioned program package and the B3LYP/6$31 \mathrm{G}(\mathrm{d})$ derived force fields. Values of the solvent dielectric constants ranging from $\epsilon=1$ (vacuum) to $\epsilon=80.1$ (water) were used, including $\epsilon=2$ (hexadecane), $\epsilon=10$ (tert-butyl alcohol) and $\epsilon=$ 35.9 (acetonitrile). 


\section{Results and discussion}

\subsection{Conformational energy differences}

Table 1 presents the values of the most relevant structural parameters for the more stable conformers of $2 \mathrm{ME}\left(\mathrm{tg} g^{\prime}, \mathrm{gg} g^{\prime}, \mathrm{ttg}, \mathrm{ttt}\right)$, and defined values of the solvent dielectric constant. Conformational energy differences $\left(\Delta E / \mathrm{kJ} \mathrm{mol}^{-1}\right)$ for these $2 \mathrm{ME}$ conformers are plotted in Fig. 1 as functions of the solvent dielectric constant. As it can be seen, all the considered $2 \mathrm{ME}$ conformers are stabilized when the solvent dielectric constant increases, since they all have dipole moments. In the considered range of $\epsilon$ (from 1 for vacuum, to 80.1 for water), $\operatorname{tgg}^{\prime}$ is stabilized ca. $22 \mathrm{~kJ} \mathrm{~mol}^{-1}, g g g^{\prime}$ by $25 \mathrm{~kJ} \mathrm{~mol}^{-1}$, ttg by $27 \mathrm{~kJ} \mathrm{~mol}^{-1}$, and $t t t$ by $24 \mathrm{~kJ} \mathrm{~mol}^{-1} \cdot \operatorname{tgg}^{\prime}$, the most stable conformer in the vacuum, is still the most stable conformer in water, and the intramolecularly hydrogen-bonded conformers, $\operatorname{tgg}^{\prime}$ and $g g g^{\prime}$, decrease in their stability relatively to the non-hydrogen-bonded conformers, ttg and ttt. These conformational energy differences vary mainly for dielectric constant values up to $\epsilon=$ 10 , remaining almost constant for higher values. Boltzmann populations decrease $9 \%$ for $\mathrm{tgg}^{\prime}$ (from $95 \%$ to $86 \%$ ), and increase $6 \%$ for $\mathrm{ggg}^{\prime}$ (from $4 \%$ to $10 \%)$ and $3 \%$ for the other two conformers.

\subsection{Conformers structures}

The following general trends can be drawn form Table 1. Starting with the alkylic part of the molecule, the $\left(\mathrm{H}_{3}\right) \mathrm{C}-\mathrm{O}$ bond length increases ca. $0.5 \mathrm{pm}$ for all the conformers when $\epsilon$ varies from 1 to 10 , stabilising for higher values of $\epsilon$. However, the bond angle C$\mathrm{O}-\mathrm{C}$ decreases $0.2^{\circ}-0.5^{\circ}$, and the dihedral angle $\mathrm{CO}-$ $\mathrm{CC}$ decreases $1^{\circ}$ in $\operatorname{tgg}^{\prime}, 1.6^{\circ}$ for $g g g^{\prime}$, and $2.2^{\circ}$ for $t$ tg.

For the intramolecularly H-bonded conformers, $\operatorname{tgg}^{\prime}$ and $\operatorname{ggg}^{\prime}$, it is worth mentioning that the contact distance between the ether $\mathrm{O}$ atom and the hydroxyl $\mathrm{H}$ atom, $\mathrm{O} \cdots \mathrm{H}(\mathrm{O})$, increases ca. $3 \mathrm{pm}$ with the increase of $\epsilon$ (Table 1).

In addition, the bond angle $\mathrm{C}-\mathrm{O}-\mathrm{H}$ increases slightly $\left(0.1^{\circ}\right)$ for $\operatorname{tgg}^{\prime}$ and $g g g^{\prime}$, and decreases by the same amount for the non H-bonded conformers. The angle $\mathrm{O} \cdots \mathrm{H}-\mathrm{O}$ decreases more significantly for $\operatorname{tgg}^{\prime}\left(1.7^{\circ}\right)$ than for $g g g^{\prime}\left(0.2^{\circ}\right)$. In turn, the dihedral angle $\mathrm{CC}-\mathrm{OH}$ increases significantly for $g g g^{\prime}\left(2.8^{\circ}\right)$ and decreases $0.6^{\circ}$ for $\operatorname{tgg}^{\prime}$.

\subsection{Charge distributions}

Charge distributions, as represented by molecular dipole moments and Mulliken atomic charges, can be compared for the studied 2ME conformers by inspection of the values presented in Table 1. In particular, the molecular dipole moment increases for all the $2 \mathrm{ME}$ conformers when $\epsilon$ varies from 1 (vacuum) to 80.1 (water): $0.41 \mathrm{D}$ for $\operatorname{tgg}^{\prime}$, corresponding to $17 \%$ of the isolated molecule value; $0.44 \mathrm{D}$ for $g g g^{\prime}, 18 \%$; $0.51 \mathrm{D}$ for $t t g, 25 \%$; $0.08 \mathrm{D}$ for $t t t, 21 \%$ ).

Passing now to the consideration of Mulliken atomic charges, the ether $\mathrm{O}$ atom becomes more negative with $\epsilon$ for all the above mentioned conformers, though by no more than $2.5 \times 10^{-2}$ charge units. In addition, the hydroxyl $\mathrm{H}$ atom becomes more positive with $\epsilon$ for all the conformers. However, in the case of the H-bonded conformers, $\operatorname{tgg}^{\prime}$ and $g g g^{\prime}$, for which the hydroxyl $\mathrm{H}$ atom is more positive than for $\mathrm{ttg}$ and tt when $\epsilon=1$, this charge increase does not exceed $2 \times 10^{-2}$ charge units. In turn, for the non $\mathrm{H}$-bonded conformers, the same charge increase exceeds $4 \times 10^{-2}$ charge units, thus making the $\mathrm{H}$ atom more positive for $t \mathrm{tg}$ and ttt than for $\mathrm{tgg}^{\prime}$ and $g g g^{\prime}$.

This behaviour points to an additional polarization for the hydroxyl group with increasing dielectric constant. For the H-bonded conformers, tgg $^{\prime}$ and $g g g^{\prime}$, the polarization is not so strong, probably because of the competition between intramolecular and solute-solvent interactions.

From the above changes in the conformers' dipole moments, it can be generally concluded that the molecular dipole moment is particularly sensitive to the solvent dielectric constant. By contrast, the Mulliken charges do not exhibit appreciable sensitivity to the solvent dielectric constant. Hence, it can be concluded that the dipole moment changes are caused by the above mentioned geometry changes rather than by redistribution of charges.

\subsection{Vibrational frequencies}

Table 1 presents the vibrational frequencies obtained with quadratic force fields for the four more stable conformers of $2 \mathrm{ME}, \mathrm{tgg}^{\prime}, \mathrm{gg} g^{\prime}, \mathrm{ttg}$ and 
$\mathrm{ttt}$, and defined values of the solvent dielectric constant ranging from 1 to 80.1 (water).

As it can be seen from this Table, the $\mathrm{OH}$ stretching frequency decreases by 7 and $11 \mathrm{~cm}^{-1}$ for $\mathrm{ttg}$ and $\mathrm{ttt}$, respectively, and varies neither monotonically nor significantly for the hydrogen-bonded conformers, $\operatorname{tgg}^{\prime}$ and $\operatorname{ggg}^{\prime}$. In turn, the $\mathrm{C}-\mathrm{O}-\mathrm{H}$ bending frequency decreases with $\epsilon$ ca. 11 and $16 \mathrm{~cm}^{-1}$ for the H-bonded conformers, $\operatorname{tgg}^{\prime}$ and $g g g^{\prime}$, respectively, and does not change significantly for the other conformers. Finally, the $\mathrm{CC}-\mathrm{OH}$ torsion exhibits a negative change in frequency of ca. $11 \mathrm{~cm}^{-1}$ for $\mathrm{ggg}^{\prime}$, and a positive change of ca. $20 \mathrm{~cm}^{-1}$ for $\mathrm{ttt}$.

\section{Conclusions}

The above mentioned results highlight changes induced by variation of the solvent dielectric constant in two kinds of molecular properties for the $2 \mathrm{ME}$ conformers, namely, geometry and dipole moments. In addition, the effect of the solvent as a polarizable continuum is not found to be strong enough to change the relative stability of the two more stable conformers which have intramolecular hydrogen bond contacts, namely, $\operatorname{tgg}^{\prime}$ and $g g g^{\prime}$. From the vibrational point of view, no significant changes were generally observed. Moreover, as the previously recorded FTIR and Raman spectra of liquid 2ME has shown features ascribed to different degrees of aggregation through intermolecular hydrogen bonding [1], it can be concluded that only specific solute-solvent interactions are likely to be vibrationally significant.

In addition, the herein obtained results seem to suggest that the intramolecular $\mathrm{H}$-bond type of interactions, which occur for $\operatorname{tgg}^{\prime}$ and $g g g^{\prime}$, tend to attenuate the solvent influence. In fact, these conformers decrease in stability relatively to the other two conformers, $t t g$ and $t t t$, although they remain the most stable conformers, with populations of $86 \%$ and $10 \%$, respectively for values of $\epsilon$ above 10 .

In turn, the conformers which do not exhibit intramolecular $\mathrm{H}$-bond interactions, $\mathrm{ttg}$ and $\mathrm{ttt}$, exhibit a stronger solvent influence as revealed mainly by structural and electrostatic changes affecting both the ether $\mathrm{O}$ atom and the hydroxyl group.

\section{Acknowledgements}

The authors thank Fundação de Ciência e Tecnologia (FCT), Lisboa, for supporting Research Units №157/94, 'Centro de Química Inorgânica e de Materiais', and $\mathrm{N}^{\circ} 30 / 94$, 'Física Nuclear e Óptica Aplicadas', where the present work was carried out.

\section{References}

[1] F.P.S.C. Gil, R. Fausto, A.M. Amorim da Costa, J.J.C. Teixeira-Dias, J. Chem. Soc. Faraday Trans. 90 (1994) 689.

[2] F.P.S.C. Gil, J.J.C. Teixeira-Dias, J. Mol. Sruct. (Theochem) 332 (1995) 269.

[3] H.S. Domingos, F.P.S.C. Gil, J.J.C. Teixeira-Dias, J. Mol. Struct. (Theochem) 401 (1997) 181.

[4] F.P.S.C. Gil, A.M. Amorim da Costa, R.E. Bruns, J.J.C. Teixeira-Dias, J. Phys. Chem. 99 (1995) 634.

[5] J.B. Foresman, T.A. Keith, K.B. Wiberg, J. Snoonian, M.J. Frisch, J. Phys, Chem. 100 (1996) 16098.

[6] J.B. Foresman, M.J. Frisch, Exploring Chemistry with Electronic Structure Methods, 2nd Edition, Gaussian, Inc, Pittsburgh, PA, 1996.

[7] Gaussian 94, Revision E.3, M.J. Frisch, G.W. Trucks, H.B. Schlegel, P.M.W. Gill, B.G. Johnson, M.A. Robb, J.R. Cheeseman, T. Keith, G.A. Petersson, J.A. Montgomery, K. Raghavachari, M.A. Al-Laham, V.G. Zakrzewski, J.V. Ortiz, J.B. Foresman, J. Cioslowski, B.B. Stefanov, A. Nanayakkara, M. Challacombe, C.Y. Peng, P.Y. Ayala, W. Chen, M.W. Wong, J.L. Andres, E.S. Replogle, R. Gomperts, R.L. Martin, D.J. Fox, J.S. Binkley, D.J. Defrees, J. Baker, J.P. Stewart, M. HeadGordon, C. Gonzalez, J.A. Pople, Gaussian, Inc., Pittsburgh, PA, 1995. 